\title{
Learning-Based Testing of Cyber-Physical Systems-of-Systems: a Platooning Study
}

\author{
Karl Meinke \\ School of Computer Science and Communication, \\ KTH Royal Institute of Technology, 10044 Stockholm, Sweden
}

\begin{abstract}
Learning-based testing (LBT) is a paradigm for fully automated requirements testing that combines machine learning with modelchecking techniques. LBT has been shown to be effective for unit and integration testing of safety critical components in cyber-physical systems, e.g. automotive ECU software.

We consider the challenges faced, and some initial results obtained in an effort to scale up LBT to testing co-operative open cyber-physical systems-of-systems (CO-CPS). For this we focus on a case study of testing safety and performance properties of multi-vehicle platoons.
\end{abstract}

Keywords: cyber-physical system, system-of-systems, platooning, model-based testing, learning-based testing, machine learning, requirements testing

\section{Introduction}

A cooperating cyber-physical system-of-systems can be characterised by the use of wireless communication, multiple stakeholders, dynamic system definitions, and unpredictable operating environments. Such systems-of-systems have been termed Cooperative Open Cyber-Physical Systems (CO-CPS) [33]. It is assumed that no single stakeholder has overall system responsibility, and that cooperation relies on wireless communication to perform safety-relevant functions.

CO-CPS are emerging around the world, due to rapid progress in telecommunications, robotics and AI. Many examples can be found in Cooperative Intelligent Transport Systems (C-ITS) and intelligent manufacturing. However, they represent a great challenge to the software quality assurance (SQA) community. Not least, the cyber-physical character of CO-CPS means that the impact of safety and security incidents (malicious or unintended) is potentially very high. However, if we survey the range of current technologies available for SQA, we can find significant limitations in many current approaches to quality assurance of CO-CPS.

On the one hand, the dynamic and heterogeneous nature of CO-CPS makes a full static analysis technically difficult. The sheer scale of many proposed COCPS suggests that a full system-of-systems analysis would even be technically infeasible. Furthermore, it is unclear (for commercial reasons) whether all source code in a CO-CPS would ever be made available for this. Static analysis of the 
individual components by their vendors might be technically feasible. However, it is difficult to see how such low-level component analysis could take into consideration unpredictable environment factors and high-level emergent phenomena (such as physical collisions). For this reason, software testing, laboratory simulations and field tests are the de-facto SQA standard used in industry today. Here the problem is that software testing traditionally focuses on unit, integration and system level testing. Simulation and field testing can be reliable and decisive at the level of systems-of-systems, but tend to be slow and unsystematic in their coverage. There is thus a great need to perform systematic and fully automated requirements testing on CO-CPS.

The scalability problem for quality assurance of CO-CPS might be made more tractable by taking a model-based approach, using judicious abstraction to suppress irrelevant technical detail. However, one is still faced with the fact that not all software vendors will take a model-driven approach, let alone exchange their models for IPR reasons. Therefore, in the worst case one would be left to perform a model based analysis where some component models are known, but others are missing, inconsistent with code, or out of date.

Against this background situation for CO-CPS, within the EU ECSEL project SafeCOP ${ }^{1}$, we are evaluating the potential of a technology known as learningbased testing (LBT) $[24,23]$. LBT is a paradigm which combines techniques from model-driven development (e.g. model-based testing, model checking of safety requirements etc) with machine learning. The basic idea is to use machine learning to reverse engineer a behavioral system model from runtime observations of a system under test (SUT). Since LBT is a black-box technique, it is code and platform independent, potentially scalable, and need not infringe upon component IPRs. The runtime SUT observations can be made either by laboratory simulation (e.g. software-in-the-loop SIL, hardware-in-the-loop HIL) or field testing. The learned model can then be used to analyse safety properties [11], and even security properties [14], by using appropriate tools such as model checkers. Potential system anomalies discovered during model analysis are classified as true negatives or false negatives by executing the corresponding test cases on the SUT.

We present here some initial results of applying LBT to a case study of testing co-operative vehicle platoons [4]. One reason for choosing this case study is because the problem size can be scaled up uniformly by adding more vehicles. This allows us to measure the influence of different factors on the scalability of LBT technology

The case study of platooning presented here is a first attempt to address two important questions about state-of-the-art LBT technology:

(1) how well does recently developed multi-core based LBT technology scale up to testing complex CO-CPS scenarios;

(2) how do problem size and other factors affect scalability?

The organisation of this paper is as follows. In Section 2 we review fundamental concepts and the state-of-the-art in learning-based testing. In Section

\footnotetext{
1 See www.safecop.eu
} 
3 , we consider the architecture and functionality of platooning as a CO-CPS. In Section 4 we present our case study of LBT applied to a platoon model. In Section 5 we survey related work in the literature. Finally in Section 6 , we draw conclusions from our intial results, and comment on future research directions.

\section{Learning-based Testing}

In this section, we review some fundamental principles of learning-based testing as these have been implemented in our research tool LBTest. The earliest version of this tool (LBTest 1.x) has been described in [26]. Therefore we will focus on the latest tool architecture LBTest 3.x, presented in Figure 1. In Section 2.1 we use this architecture to explain the basic principles of LBT. Then, in Sections 2.2 and 2.3, we show how concurrent aspects of this architecture contribute towards solving tool scalability issues ${ }^{2}$.

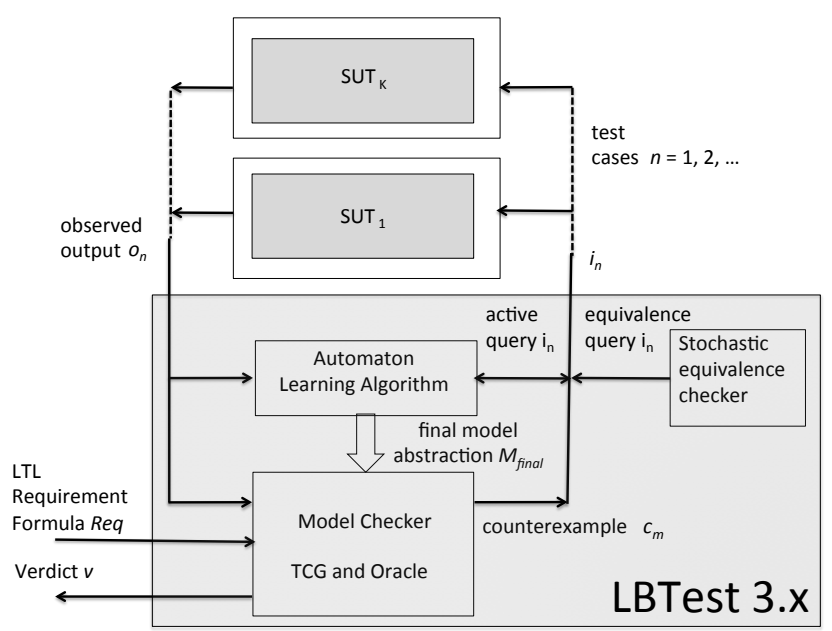

Fig. 1. LBTest 3.x concurrent learning architecture

\subsection{Principles of LBT}

LBTest uses active automaton learning aka. regular inference (see e.g. [13]) to generate queries about a black-box system, which can be used to construct a behavioral model in polynomial time, modulo an equivalence oracle [2].

\footnotetext{
2 This architecture has been developed within the VINNOVA FFI project VIRTUES, http://www.csc.kth.se/ karlm/virtues/
} 
For requirements testing, partial models of the SUT can be subjected to model checking against a temporal logic requirement specification, even before the learning process is complete. In LBTest, propositional linear temporal logic ${ }^{3}$ (PLTL) is used as a requirements modeling language. This particular logic has the advantage that test cases can easily be extracted from counterexamples generated by a model checker. LBTest makes use of a loosely integrated symbolic checker NuSMV [7]. We are also developing a more tightly integrated explicit state model checker for efficiency reasons. These two processes of learning and model checking may be interleaved, an idea first suggested in [27]. Then they incrementally build up a sequence $M_{1}, M_{2}, \ldots$ of models of the SUT, while generating and executing requirements test cases on each model $M_{i}$.

To separate true negatives (SUT errors) from false negatives (artifacts of an incomplete model) it is necessary to validate each counter-example derived from model checking. For this we can: (i) extract a test case representing the counterexample $^{4}$, (ii) execute it on the SUT, (iii) apply an equality test that compares the observed SUT behavior with the predicted bad behavior from the model, and (iv) automatically generate the test verdict (pass, fail) from step (iii). The soundness of this process relies on the soundness of the underlying model checker, and the soundness of equality testing.

The completeness of LBT relies on the completeness of the underlying model checker, as well as convergence results about the learning algorithms which are used (see [13]). However, within practical case studies of large complex systems it may not be possible for learning to converge in any reasonable time frame (see e.g. [11]). This problem is significant for CO-CPS. Therefore, development of LBTest has focused on incremental learning algorithms that can generate incomplete approximating models of the SUT in small increments.

One measure of the coverage achieved by LBT is in terms of the behavioral accuracy of the final model. This accuracy could be defined in terms of trace inclusion between the model and the SUT. However, phenomena of both over and under approximation often occur within the same partial model, i.e. no strict trace inclusion holds either way. Nevertheless, by using a probably exactly correct (PEC) model of convergence, we can obtain convergence measures that increase monotonically over time.

Figure 1 illustrates the stochastic equivalence checker used in LBTest 3.x. This checker empirically estimates the behavioral accuracy of the final learned model $M_{\text {final }}$ for replicating the behavior of the SUT on a randomly chosen set of input sequences. For this, the input sequences are executed both on the SUT and the model. We then measure the percentage of behaviorally identical output sequences generated by both. This convergence model is stricter than the PAC convergence model of [30], since the degree of behavioral identity is estimated.

\footnotetext{
${ }^{3}$ Recall that propositional LTL extends basic propositional logic with the temporal modalities $\mathrm{G}(\phi)$ (always $\phi$ ), F $(\phi)$ (sometime $\phi$ ) and $\mathrm{X}(\phi)$ (next $\phi$ ). Other derived operators and past operators may also be included. See e.g. [12] for details.

${ }^{4}$ Infinite counter-examples to LTL liveness formulas are truncated around the loop, and the weaker test verdict warning may be issued.
} 
PEC convergence seems well suited to the needs of software safety analysis over the discrete data type partitions commonly employed in testing.

\subsection{Towards Scalable LBT Architectures}

From empirical studies such as $[11,20,25]$ we have observed two important obstacles to scaling up LBT methods for large and complex SUTs. These are:

(i) the tendency for learned model size to increase rapidly with SUT size;

(ii) the tendency for test latency (i.e. the time to execute a single test case) to increase with SUT size.

Even worse, these two problems compound one another, leading to long test session times and low final convergence measures. In benchmarking the architectural proposal of [27] we have also observed another significant problem:

(iii) model checking each member $M_{i}$ of a converging sequence of models $M_{1}, M_{2}, \ldots$ is highly inefficient, and does not seem to improve the rate of model convergence.

We will consider each of these issues, and how it can be addressed, in turn.

(i) Model Size The size of a learned model is a function of the code complexity of the underlying SUT, as well as the number of parameters of the SUT which the learning algorithm tries to stimulate and observe.

One factor influencing model size is the number of SUT input variables and the number of test values chosen for each input variable. These parameters bound the number of exit transitions from each model state. The number of exit transitions is further influenced by the combinatorial strategy used to generate composite input test vectors from the individual input variable values. A judicious combinatorial choice is necessary to control the otherwise exponential explosion in the number of transitions. In LBTest 3.x, $n$-wise testing [17] is available as a combinatorial strategy.

Another factor influencing model size is the number of observed SUT output variables, and the number of output value partition classes for each output variable. These factors influence the number of states in a learned model, since more output variables and finer output partitions lead to more easily distinguished SUT states.

So, a judicious choice of model accuracy, combinatorial test strategy and model abstraction can all be applied to improve the efficiency of learning and testing.

Besides these test configuration parameters, the problem of large model sizes has also been ameliorated by new research into machine learning algorithms. Since Angluin's seminal algorithm [2], many new learning algorithms, that can learn a model with fewer and/or shorter queries, have been derived, e.g. [16].

(ii) Test Latency Improvements in learning and model checking algorithms are scarcely able to overcome a distinctive feature of large complex SUTs which is the tendency towards long test latency times. For CO-CPS, communication 
network delays also become significant. Test latency times can become a significant component of the test session length.

To address this issue, we have been conducting research into parallelized learning algorithms on multi-core platforms. Already in [15] certain improvements in learning performance by parallelization have been reported. An important challenge is to systematically characterize such improvements in terms of problem size parameters. Our work contributes to this area by studying a parameterized and uniformly scalable learning problem namely platooning. As the size (i.e. number of vehicles) of a platoon of identical vehicles scales up, the problem parameters:

(i) total number of lines of code under test, and

(ii) total number of program registers determining the global state space, both increase linearly. Thus it becomes meaningful to compare testing results for different platoon sizes (c.f. the similar curves in Figure 4). Without such uniform properties, benchmark results across an ad-hoc collection of SUTs can be very difficult to interpret.

(iii) Model Checking Overheads Incremental learning generates a convergent sequence of models $M_{1}, M_{2}, \ldots$. However, each model $M_{i}$ will contain a good many structural features (states and transitions) that persist in model $M_{i+1}$. It is beyond the capability of any model checker we know of to identify these persistent features and avoid checking them twice in both $M_{i}$ and $M_{i+1}$. Therefore, a long model generation sequence will contain significant redundant model checking effort. Our empirical observations with LBTest 2.x and NuSMV have shown that this redundant checking can consume more than $50 \%$ of the overall test session time. Furthermore, as reported in [21], model checker generated queries have not been observed to accelerate the convergence of learning in any case study so far. ${ }^{5}$

While it might be possible to introduce a sophisticated delta-oriented approach to model checking, the simplest solution seems to be to defer model checking until after machine learning.

\subsection{Concurrent Multi-core LBT}

Figure 1 illustrates a new architecture for LBT that significantly departs from the proposals of $[23,27]$. Two new features are prominent, and both are intended to counter the scalability bottlenecks described in Section 2.2.

Firstly, the new architecture supports parallel execution of multiple instantiations of the SUT on a multi-core platform. The aim is to mitigate long SUT test latency. At the start of a test session, LBTest clones $K$ versions of the executable SUT, each within its own external OS process. The value of $K$ is chosen as a function of the number of SUT input values to be tested. Once started,

\footnotetext{
${ }^{5}$ It seems possible to theoretically explain this observation for certain types of formulas by considering their semantics. However, this is outside the scope of our present discussion.
} 
each SUT process persists throughout the learning phase, and acts as a server to answer certain kinds of queries about SUT behavior. Different load balancing schemes on these query servers are used according to the learning strategy.

Of course, concurrent execution is a rather obvious solution to test latency. The real technical challenge here is to devise efficient parallel learning algorithms that can allow multiple threads to efficiently and safely perform concurrent updates on a single shared automaton model. At the same time we need to optimise multi-core usage on the hardware level. For this we have investigated concurrent implementations of Kearn's algorithm [19]. For reasons of space, these rather complex concurrent algorithms will be described elsewhere.

The second new feature of LBTest 3.x is its support for deferred model checking, as described in Section 2.2, using an iteration bound to terminate learning. Only when learning is terminated do model checking and counter-example validation of the final model $M_{\text {final }}$ begin in a second phase. This minimises the redundant model checking identified in Section 2.2.

\section{Platooning as a CO-CPS}

In this Section we review some general features of platooning that characterise it as a CO-CPS. Then we discuss the particular platooning model that was tested in Section 4.

\subsection{General Principles of Platooning}

Platooning technology is sometimes called an "electronic towbar" between road vehicles, and this phrase gives much insight into the idea.

A platoon consists of a sequence of road vehicles $V_{1}, \ldots, V_{n}$ which (by means of sensors, wireless $\mathrm{V} 2 \mathrm{~V}$ communication and control algorithms for longitudinal or distance control) are able to maintain a fixed distance $x_{r}$ between one another and a relative velocity $v_{r}=0$ under normal cruising conditions. (See Figure 2, adapted from [5].) The lead vehicle, $V_{1}$, is under manual control by a qualified platoon leader who needs to have the necessary technical skills to control the platoon. The vehicles $V_{2}, \ldots, V_{n}$ are its followers, and may be autonomous or semiautonomous, depending on the extent to which lateral control (i.e. steering) is automated.

A platoon may be heterogeneous, consisting of different models from different vendors carrying different payloads. It should be possible to add and remove vehicles dynamically during a journey, and there are many safety critical use cases, such as lane change, emergency braking etc.

The interest in platooning technology, lies in the possibility to reduce fuel consumption and corresponding CO2 emissions, as well as to improve road usage and safety while reducing traffic congestion (see e.g. [29]). Platoons exploit the reduced aerodynamic drag that arises with short inter-vehicle distances. There is an important trade-off between fuel efficiency and safety in platoon design, since drag is reduced by shorter inter-vehicle spacing. System response times, 


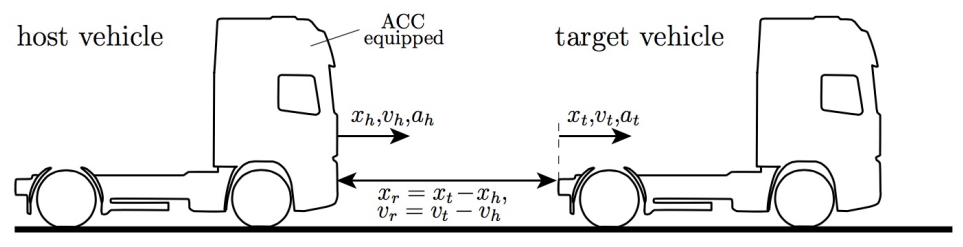

Fig. 2. Platoon Vehicle Pair: $V_{i+1}$ (left) and $V_{i}$ (right)

component reliability, road hazards and the effects of safety critical uses cases such as emergency braking on the platoon and its environment all need to be evaluated during software design.

\subsection{A Simple Platooning Model}

For pragmatic reasons, our study of LBT scalability was restricted to softwarein-the-loop (SIL) testing of a basic platoon simulator. The simulation is 1dimensional, meaning that no steering model is used. The simulator is therefore only able to analyze certain use cases, such as straight-line cruising and emergency braking. Other use cases need a more complex simulation model, and this is the subject of ongoing research and industrial collaboration. However, our model includes many important physical characteristics such as maximum engine and brake torque, vehicle mass, aerodynamic drag etc. defined using a point-mass modeling approach. (See e.g. [34] for an introduction to vehicle modeling.)

The simulator consists of about 2000 Java LOC. However, to get a clearer impression of the underlying SUT complexity we provide here some details about its structure and function.

The block architecture of a single vehicle in the platoon simulator is illustrated in Figure 3. This depicts a brake-by-wire BBW subsystem augmented with a co-operative adaptive cruise controller CACC. The latter is connected to an odometry unit ODOM (providing host vehicle position and velocity) and a wireless communication WCOM unit (relaying host and target positions and velocities). Odometry is based upon host velocity measurements ${ }^{6}$. The WCOM unit simulates a $2 \mathrm{~ms}$ inter-vehicle wireless message delay, without any transmission error model.

The CACC controller is a crucial component that provides longitudinal control of each follower vehicle. It dynamically issues accelerator and brake torque requests to maintain the position of the host vehicle within maximimum and minimum distances from the target vehicle in front. A wide variety of CACC algorithms have been proposed in the literature. The controller tested here is a simple PD control algorithm with adaptive parameters, taken from [5]. For a

\footnotetext{
${ }^{6}$ In practise, GPS localisation would be relied upon for greater accuracy.
} 


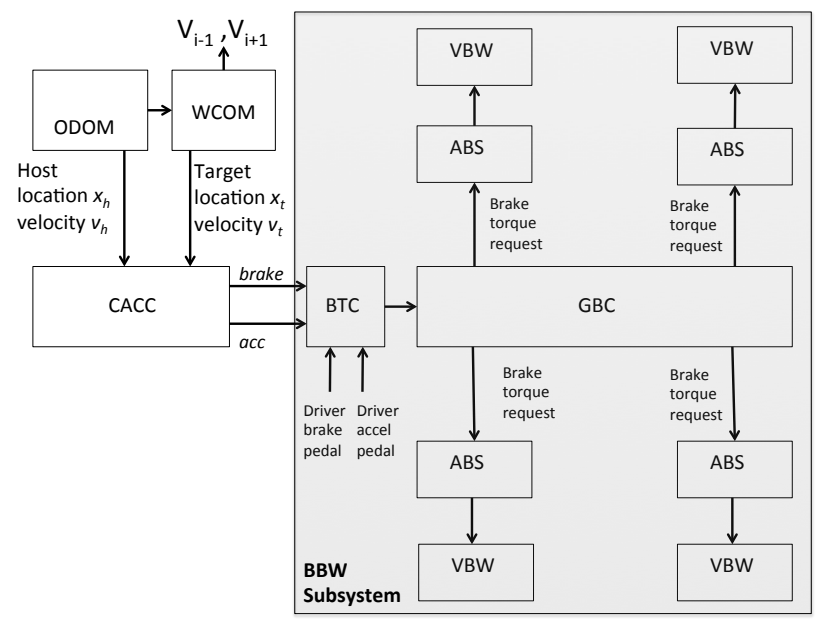

Fig. 3. Software architecture for platoon vehicle $V_{i}$

general introduction to PID control theory one may consult e.g. [10]. The function of any PID controller in the context of an ACC problem is to maintain the relative position of the host vehicle $V_{i+1}$ within the boundaries $x_{r, d, \max }$ and $x_{r, d, \min }$ (metres) from the target $V_{i}$, where

$$
x_{r, d, \max }=t_{h w} \cdot v_{h}+x_{r, 0}, \quad x_{r, d, \min }=\left(t_{h w}-t_{h w, \delta}\right) \cdot v_{h}+x_{r, 0} .
$$

Here $t_{h w}$ (seconds) is the time headway between $V_{i+1}$ and $V_{i}$, and $t_{h w, \delta}$ causes a small difference in headway. The parameter $x_{r, 0}>0$ (metres), maintains a safe relative inter-vehicle distance at $v_{h}=0$ (metres/second), to support so called stop-and-go functionality. The host position is maintained by two PD equations:

$$
\begin{gathered}
\text { acc }=K_{A C C}\left(k_{x_{r}} \cdot\left(x_{r}-x_{r, d, \max }\right)+k_{v_{r}} \cdot v_{r}\right), \\
\text { brake }=K_{A C C}\left(k_{x_{r}} \cdot\left(x_{r}-x_{r, d, \min }\right)+k_{v_{r}} \cdot v_{r}\right),
\end{gathered}
$$

governing requested accelerator and brake torque. In the above formulas: (i) $K_{A C C}$ (dimensionless) is a constant overall gain parameter. (ii) $x_{r}=x_{t}-x_{h}$ (metres) and $v_{r}=v_{t}-v_{h}$ (metres/second) are the relative distance and velocity to the target vehicle (c.f. Figure 2). (iii) $k_{x_{r}}$ is the $\mathrm{P}$ action: this gain is tuned to regulate the distance error to zero $\left(x_{r}-x_{r, d, \max }=0\right.$ for $a c c$ and $x_{r}-x_{r, d, \min }=0$ for brake). (iv) $k_{v_{r}}$ is the $\mathrm{D}$ action and the regulated error is $v_{r}$. (v) Since acc is smaller than brake (due to a different desired distance), it takes some time before the brakes are activated after the accelerator is released. 
In this PD controller design, $k_{x_{r}}$ and $k_{v_{r}}$ are dimensionless adaptive parameters:

$$
k_{x_{r}}=k_{x_{r}, 1}\left(v_{h}\right) \cdot k_{x_{r}, 2}\left(x_{r}-x_{r, d, \max }\right), \quad k_{v_{r}}=k_{v_{r}}\left(x_{r}-x_{r, d, \max }\right) .
$$

All forces acting on the vehicle, both positive and negative, are resolved at each wheel individually.

To inject behavioral faults into our platooning model for testing, we replaced the non-linear adaptive parameter functions $k_{x_{r}, 1}, k_{x_{r}, 2}, k_{v_{r}}: \mathbb{R} \rightarrow \mathbb{R}$ of [5] with highly simplified piecewise linear approximations. These linear approximations to non-linear functions make the brake and accelerator control responses, acc and brake, less smooth with both over- and under-compensation for change, as we show in Section 4.2.

For each follower vehicle, the BBW subsystem takes the accelerator and brake torque requests from $\mathrm{CACC}$, and translates these into forces on the four vehicle body wheels $\mathrm{VBW}^{7}$. The brake torque controller BTC calculates the global brake torque request (in Newton metres)

$$
\text { torqueRequest }=(\text { brake/100).maxBrakeTorque }
$$

and the global brake controller GBC distributes this brake request to each antilocking brake system $A B S_{i}$, which controls wheel $V B W_{i}$.

The fundamental simulation cycle corresponds to $1 \mathrm{~ms}$ of real-world time, while the various architectural components have execution cycle times varying between 2 and $20 \mathrm{~ms}$. Normally, vehicle software components would communicate periodically (but not necessarily deterministically) using the vehicle's CAN bus network, while the vehicles themselves communicate asynchronously. However, it is common industrial practise to perform SIL testing using a simplified synchronous composition of components to ensure reproducibility of test results. So our platoon simulator is also based on a synchronous composition of all architectural components, as well as the platoon vehicles themselves.

\section{Test Experiment Design and Results}

In this section, we first describe our testing experiment conducted on the platooning simulator described in Section 3, using the LBT tool architecture described in Section 2.3. We then describe the test results obtained, and interpret these from the perspective of LBT scalability.

\subsection{Test Experiment Design}

To test the primary use case of high-speed cruising for a platoon configuration of $n$ vehicles, we focused on emulating the lead driver behavior, since in our

\footnotetext{
${ }^{7}$ For the lead vehicle, CACC is disabled and accelerator and brake pedal values are used by BBW instead. See Figure 3.
} 
simulator all follower vehicles autonomously adapt to this. Thus, each test case $t c$ for an $n$-vehicle platoon consisted of a sequence $t c=\left(r_{1}, r_{2}, \ldots, r_{\lambda}\right)$ of lead driver accelerator and brake torque requests $r_{j}$. The continuous input spaces for each of these two input variables (accelerator and brake pedal angles) were sampled at $10 \%$ intervals, yielding $K=21$ symbolic input values $0, a_{1}, \ldots, a_{10}, b_{1}, \ldots, b_{10}$ ranging from $0 \%$ to $100 \%$ pedal depression ${ }^{8}$. No assumptions were made about lead driver behavior, so both excessive and sporadic acceleration and braking could occur. The time headway $t_{h w}$ between each successive pair of vehicles was nominally set to 2.0 seconds. A time headway of this size is normally quite safe for commercial CACC algorithms (see e.g. [5]).

For each test case $t c=\left(r_{1}, r_{2}, \ldots, r_{\lambda}\right)$, the length $\lambda$ and torque requests $r_{j}$ were chosen dynamically both by the learning algorithm and the equivalence checker. In the experiments of Section 4.2, $\lambda$ typically took an average value around 12 . The test case $t c$ was then submitted to one of $K=21$ SUT server processes $S_{p}$ executing an $n$ vehicle platoon simulator instance. The communication wrapper around $S_{p}$ loaded and executed the request sequence $\left(r_{1}, r_{2}, \ldots, r_{\lambda}\right)$ sequentially. Each torque request value $r_{j}$ was maintained constantly for a nominal 5 seconds (5000 simulation cycles). Thus the length of the simulation corresponding to $t c$ was $5 \lambda$ virtual seconds. The values chosen for $\lambda$ were sufficient to reach high cruising speeds, in excess of $110 \mathrm{Km} / \mathrm{hr}$.

Maintaining the torque request over a fixed number of seconds is a temporal abstraction technique necessary to achieve a balance between long simulation times and small final model size. This abstraction can be adjusted in the simulator. It also has the advantage that we can easily calculate the cumulative virtual simulation time for an entire test session.

The principle SUT output recorded for the test case $t c$ was the time sequence of inter-vehicle gaps $x_{r, 0}^{i}, \ldots, x_{r, \lambda}^{i}$, for each vehicle $i=1, \ldots n-1$. Here, the time sequence term $x_{r, t}^{i}$, for $0 \leq t \leq \lambda$, represents the gap between the host-target pair, $V_{i}$ and $V_{i+1}$ measured at the end ${ }^{9}$ of $5 t$ virtual seconds (i.e. $5000 t$ simulation cycles). The continuous values of each distance observation $x_{r, t}^{i}$ were partitioned within the communication wrapper into three discrete equivalence classes:

tooClose, toofar, good,

based on the (host velocity dependent) distance boundaries $x_{r, d, \min }^{i}$ and $x_{r, d, \max }^{i}$. Thus the symbolic output good for $x_{r, t}^{i}$ represented the output partition class $x_{r, d, \min }^{i} \leq x_{r, t}^{i} \leq x_{r, d, \max }^{i}$.

To gain further insight into the physical state space covered by testing we also observed the lead vehicle velocity values $v_{0}^{1}, \ldots, v_{\lambda}^{1}$ and acceleration values $a_{0}^{1}, \ldots, a_{\lambda}^{1}$ at the same observation times. These continuous valued observations were partitioned into $1 \mathrm{Km} / \mathrm{hr}$ and $1 \mathrm{Km} / \mathrm{hr} r^{2}$ equivalence classes.

8 Thus $a_{10}$ represents $100 \%$ accelerator depression, $a_{9}$ represents $90 \%$ depression, etc. Simultaneous depression of both pedals is handled as a brake request by the BBW component.

${ }^{9}$ It is also possible to use SUT observations between the output cycles by thresholding. This can yield greater accuracy, but this approach was not taken here. 
With regard to system-of-systems requirements, the most fundamental requirement is that all $n$ platoon vehicles should always maintain a safe but fuel efficient distance between each other. This test requirement could be represented in PLTL for an $n+1$-vehicle platoon (where $n \geq 1$ ) by the safety formula:

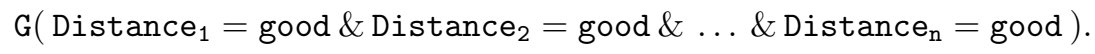

Here Distance ${ }_{i}$ represents the discretized gap between vehicles $V_{i}$ and $V_{i+1}$ corresponding to measurements $x_{r, t}^{i}$.

One experimental goal was to try to observe the injected errors in the CACC component, (described in Section 3.2) as violations of the test requirement $\left(^{*}\right.$ ). The other goal was to characterise the scalability of the tool.

\subsection{Test Experiment Results}

The test experiment described in Section 4.1 was conducted for platoon sizes $n=2, \ldots, 6$ to investigate the scalability of the testing tool. To uniformise the results, each platoon vehicle in each configuration had identical physical parameters ${ }^{10}$. We measured the final model size for different platoon sizes and different test session durations. While test session duration is a platform dependent measurement ${ }^{11}$, it was felt that this value gave good insight into tool usability and potential future improvements.

Figure 4 shows the growth of model size over time for platoon sizes $n=$ $2, \ldots, 6$ using concurrent learning. To analyse the benefit of concurrency, Figure 4 also shows model growth for $n=3$ under sequential learning. Note that the $\mathrm{y}$ axis is in thousands of states (Kstates). The graph shows the effects of increasing test latency as the platoon size increases. The largest inferred model (for $n=6$ ) had over 64,600 states and 1.35 million transitions achieved after 20 hours and 25 minutes of learning. During this time, 1.5 million test cases $t c$ were executed, with an average test case length of $\lambda=10.6$. Since each step in $t c$ corresponds to 5 virtual seconds, the total virtual testing time was over 22,000 hours.

Notable in Figure 4 is the gradual slowdown in rates of model growth over time. However, there is no sharp fall in tool performance. Furthermore, the vertical intervals between the curves are very similar, both for increasing $n$ and $t$. These two characteristics seem to suggest good scalability properties for our approach as a function of the problem size $n$.

With regard to requirements errors, NuSMV developed a segmentation fault already with the smallest of our models for $n=2$ (8826 states, $185 \mathrm{~K}$ transitions). However, using our explicit state model checker on the largest model for $n=6$ (64,671 states, 1.35 million transitions), the error tooFar was found to occur in 50,076 states ( $77 \%$ of all states), while the error tooClose was found in just 101

$\overline{10}$ Non-homogeneous platoons could also be tested using our approach.

11 The actual platform used was a 4-core MacBook Pro, Mid 2014, running Yosemite OS-X 10.10.5 with 2.8GHz Intel Core i7, 16GB $1600 \mathrm{MHz}$ DDR3 and 1TB static disk flash storage. 


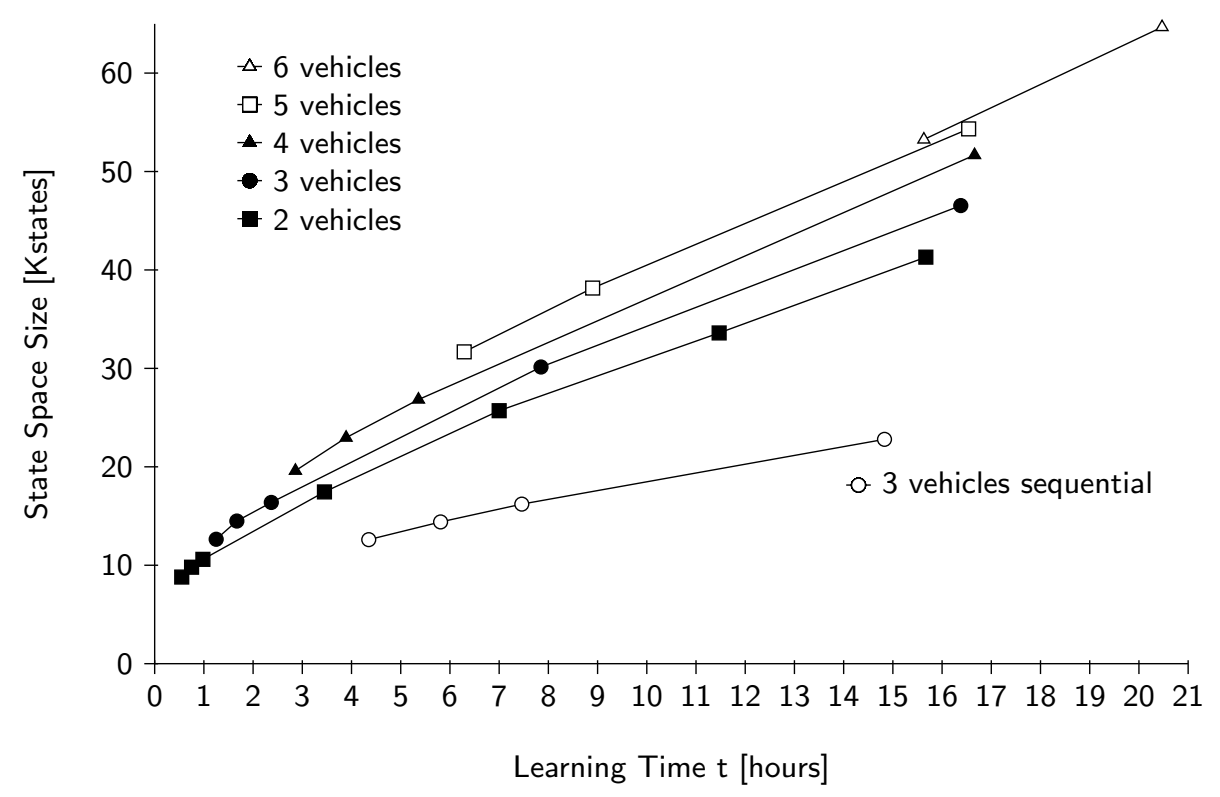

Fig. 4. Rates of model growth (state space size) over time for different platoon sizes.

states $(0.0015 \%$ of all states) after 32.4 seconds of model checking. There were no false negatives among these counter-examples. The error tooClose was found only at low velocities, mainly at $v^{1}=0$, which seems to confirm the thesis of [5] that stop-and-go functionality is rather difficult to implement correctly. For the smallest model of $n=2$ (8826 states), the error tooFar could also be found after $19 \mathrm{~ms}$ of model checking, but not error tooClose.

Through runtime monitoring, we estimated long term multi-core usage to range between $85 \%-95 \%$ over the problem size range $n=2, \ldots, 6$, with approximately $10 \%$ fluctuations short term ${ }^{12}$. At peak core usage, CPU idle time was less than $1 \%$, implying that further cores would have been of benefit.

For the experiments described in Figure 4, the platoon models reached maximum convergence values of $9.4 \%, 9.4 \%, 8.8 \%, 7.1 \%$ and $6.0 \%$ for $n=2, \ldots, 6$ respectively.

\section{Related Work}

The application of machine learning to testing has a somewhat long history, beginning with [32]. The architecture used in LBTest 2.x first appeared in [27] and was independently proposed in [24]. However, scalability and the effect of model checking on convergence, were not originally considered. Recently, the literature on machine learning applied to software engineering has become quite extensive.

\footnotetext{
$\overline{12}$ Based on 1 second sampling.
} 
Known techniques use models based on deterministic automata [28,31,23,16,14], non-deterministic finite automata [21], and extended finite state machines [6]. The emphasis ranges from unit and integration testing to software documentation. A state-of-the-art survey is [3]. Our experience [22] suggests that machine learning of hybrid automata would be too slow to deal with complex continuous state SoS such as platoons.

To our knowledge, only one other study of parallelized machine learning for testing exists, namely [15]. This shares our premise that parallel learning is important to mitigate test latency. However, it evaluates only synthetic SUT latency obtained by inserting a $5 \mathrm{~ms}$ busy waiting loop into each SUT call. Model checking and requirements testing are not considered. The authors investigate speedup of learning randomly generated SUTs of different state space sizes in the range $1, \ldots, 256$ states. They conclude that under an increasing number of cores, a saturation point is met, where adding more cores yields no benefit ${ }^{13}$. By contrast, we have varied a much larger problem size $8 K, \ldots, 64 K$ states, keeping the core number fixed.

Platooning has been widely studied in the C-ITS literature. A survey of platooning research is [4]. An account of traditional SIL and HIL testing of a 3 vehicle platooning system is [1]. This work has very similar safety concerns to our own. Examples of static analysis applied to platooning are $[8,9]$ and [18] where it is shown that verifying vehicle code does not scale to the whole systemof-systems, and a mixed top-down and bottom up strategy are applied.

\section{Conclusions and Future Work}

We have presented an initial assessment of the scalability of multi-core learningbased testing technology to cyber-physical systems-of-systems (CO-CPS). For this we have conducted testing experiments on a vehicle platooning simulator, where we have injected faults that violate safety and fuel efficiency requirements. Extensive testing experiments over different platoon sizes have demonstrated that learned model size scales well over the experimental time horizon and different platoon sizes. However, unsurprisingly perhaps, model convergence is low, at least according to the current PEC metric. Nevertheless effective testing, capable of finding both common and rare errors without false negatives, was possible by learning large but incomplete models.

Future research needs to address several issues. Learning efficiency needs to be further improved to enhance coverage. Our study could be generalized by using more advanced simulators to test other use cases. We will also further consider how to scale up LBT to many-core platforms. Can the saturation effects cited in [15] be observed or avoided? The reliability questions surrounding incomplete model learning warrant further attention, e.g. the optimal choice of a learning convergence metric is an open question. Finally, equation $\left(^{*}\right)$ of Section

${ }^{13}$ Unfortunately our limited computing platform did not provide an opportunity to evaluate this result. 
4.1 represents a safety requirement that could be captured by a suitable spatiotemporal logic. Further study of spatio-temporal logics and model checking might be fruitful for CO-CPS use case testing.

This project has received funding from the Electronic Component Systems for European Leadership Joint Undertaking under grant agreement No 692529.

\section{References}

1. Aki, M., Zheng, R., Yamabe, S., Nakano, K., Suda, Y., Suzuki, Y., Ishizaka, H., Kawashima, H., Sakuma, A.: Safety testing of an improved brake system for automatic platooning of trucks. Int. J. Intelligent Transportation Systems Research 12(3), 98-109 (2014)

2. Angluin, D.: Learning regular sets from queries and counterexamples. Inf. Comput. 75(2), 87-106 (Nov 1987)

3. Bennaceur, A., Giannakopoulou, D., Hähnle, R., Meinke, K.: Machine learning for dynamic software analysis: Potentials and limits (dagstuhl seminar 16172). Dagstuhl Reports 6(4), 161-173 (2016)

4. Bergenhem, C., Shladover, S., Coelingh, E., Englund, C., Shladover, S., Tsugawa, S.: Overview of platooning systems. In: Proc. 19th ITS World Congress, Vienna, Austria (October 2012)

5. van den Bleek, R.: Design of a Hybrid Adaptive Cruise Control Stop-\&-Go system. Master's thesis, Technische Universiteit Eindhoven, Department of Mechanical Engineering (2007)

6. Cassel, S., Howar, F., Jonsson, B., Steffen, B.: Active learning for extended finite state machines. Formal Asp. Comput. 28(2), 233-216 (2016)

7. Cimatti, A., Clarke, E., Giunchiglia, E., Giunchiglia, F., Pistore, M., Roveri, M., Sebastiani, R., Tacchella, A.: NuSMV 2: An OpenSource Tool for Symbolic Model Checking, pp. 359-364. Springer (2002)

8. Colin, S., Lanoix, A., Kouchnarenko, O., Souquieres, J.: Using CSPIIb Components: Application to a Platoon of Vehicles, pp. 103-118. Springer (2009)

9. El-Zaher, M., Contet, J., Gruer, P., Gechter, F., Koukam, A.: Compositional verification for reactive multi-agent systems applied to platoon non collision verification. Stud. Inform. Univ. 10(3), 119-141 (2012)

10. Engelberg, S.: A Mathematical Introduction To Control Theory. Imperial College Press (2015)

11. Feng, L., Lundmark, S., Meinke, K., Niu, F., Sindhu, M.A., Wong, P.Y.H.: Case Studies in Learning-Based Testing, pp. 164-179. Springer (2013)

12. Fisher, M.: An Introduction to Practical Formal Methods Using Temporal Logic. Wiley Publishing (2011)

13. De la Higuera, C.: Grammatical inference: learning automata and grammars. Cambridge University Press (2010)

14. Hossen, K., Groz, R., Oriat, C., Richier, J.: Automatic model inference of web applications for security testing. In: Seventh IEEE International Conference on Software Testing, Verification and Validation, ICST 2014 Workshops Proceedings. pp. 22-23 (2014)

15. Howar, F., Bauer, O., Merten, M., Steffen, B., Margaria, T.: The teachers' crowd: The impact of distributed oracles on active automata learning. In: Leveraging Applications of Formal Methods, Verification, and Validation - International Workshops, SARS 2011 and MLSC 2011, Revised Selected Papers. pp. 232-247 (2011) 
16. Isberner, M., Howar, F., Steffen, B.: The TTT algorithm: A redundancy-free approach to active automata learning. In: Runtime Verification - 5th International Conference, RV 2014, Proceedings. pp. 307-322 (2014)

17. Jorgensen, P.C.: Software testing (2008)

18. Kamali, M., Dennis, L.A., McAree, O., Fisher, M., Veres, S.M.: Formal verification of autonomous vehicle platooning. CoRR abs/1602.01718 (2016)

19. Kearns, M., Vazirani, U.: An Introduction to Computational Learning Theory. MIT Press (1994)

20. Khosrowjerdi, H., Meinke, K., Rasmusson, A.: Automated behavioral requirements testing for automotive ecu applications. In: Proc. 5th Int. Workshop on Model Based Safety Analysis (IMBSA 2017) - to appear. Springer LNCS (2017)

21. Meinke, K.: Recent progress in learning-based testing. In: Bennaceur, A., Hähnle, R., Meinke, K. (eds.) Machine Learning for Dynamic Software Analysis: Potentials and Limits: Proceedings of Dagstuhl Workshop 16172 (to appear). Springer (2017)

22. Meinke, K., Niu, F.: An incremental learning algorithm for hybrid automata (2013)

23. Meinke, K., Sindhu, M.A.: Incremental learning-based testing for reactive systems. In: Gogolla, M., Wolff, B. (eds.) Tests and Proofs: 5th International Conference, TAP 2011, Proceedings. pp. 134-151. Springer (2011)

24. Meinke, K.: Automated black-box testing of functional correctness using function approximation. In: Proceedings of the 2004 ACM SIGSOFT International Symposium on Software Testing and Analysis. pp. 143-153. ISSTA '04, ACM Press (2004)

25. Meinke, K., Nycander, P.: Learning-based testing of distributed microservice architectures: Correctness and fault injection. In: Bianculli, D., Calinescu, R., Rumpe, B. (eds.) Software Engineering and Formal Methods: SEFM 2015 Collocated Workshops: ATSE, HOFM, MoKMaSD, and VERY*SCART. Revised Selected Papers. pp. 3-10. Springer (2015)

26. Meinke, K., Sindhu, M.A.: Lbtest: A learning-based testing tool for reactive systems. In: Proceedings of the 2013 IEEE Sixth International Conference on Software Testing, Verification and Validation. pp. 447-454. ICST '13, IEEE Computer Society (2013)

27. Peled, D.A., Vardi, M.Y., Yannakakis, M.: Black box checking. In: Formal Methods for Protocol Engineering and Distributed Systems, FORTE XII / PSTV XIX'99, IFIP TC6 WG6.1. pp. 225-240 (1999)

28. Raffelt, H., Steffen, B., Margaria, T.: Dynamic Testing Via Automata Learning, pp. 136-152. Springer (2008)

29. Sjöberg, K.: Platooning - challenges and opportunities (2016), https: //docbox.etsi.org/Workshop/2016/201603_ITS_WORKSHOP/S04_TWDS_ ACCIDENT_FREE_AUTOMATED_DRIVING

30. Valiant, L.G.: A theory of the learnable. Commun. ACM 27(11), 1134-1142 (Nov 1984)

31. Walkinshaw, N., Bogdanov, K., Derrick, J., Paris, J.: Increasing Functional Coverage by Inductive Testing: A Case Study, pp. 126-141. Springer (2010)

32. Weyuker, E.: Assessing test data adequacy through program inference. ACM Trans. Program. Lang. Syst 5(4), 641-655 (1983)

33. Willke, T., Tientrakool, P., Maxemchuk, N.: A survey of inter-vehicle communication protocols and their applications. IEEE Communications Surveys and Tutorials $11(2), 3-20(2009)$

34. Özguner, U., Acarman, T., Redmill, K.: Autonomous Ground Vehicles. Artech House Publishers, Boston, Mass., USA (2011) 